\title{
FGF receptors are required for proper axonal branch targeting in Drosophila
}

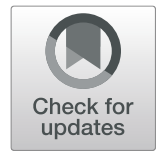

Júnia Vieira dos Santos ${ }^{1}$, Renee Yin Yu ${ }^{1}$, Andrea Terceros $^{1}$ and Brian Edwin Chen ${ }^{1,2^{*}}$ (D)

\begin{abstract}
Proper axonal branch growth and targeting are essential for establishing a hard-wired neural circuit. Here, we examined the role of Fibroblast Growth Factor Receptors (FGFRs) in axonal arbor development using loss of function and overexpression genetic analyses within single neurons. We used the invariant synaptic connectivity patterns of Drosophila mechanosensory neurons with their innate cleaning reflex responses as readouts for errors in synaptic targeting and circuit function. FGFR loss of function resulted in a decrease in axonal branch number and lengths, and overexpression of FGFRs resulted in ectopic branches and increased lengths. FGFR mutants produced stereotyped axonal targeting errors. Both loss of function and overexpression of FGFRs within the mechanosensory neuron decreased the animal's frequency of response to mechanosensory stimulation. Our results indicate that FGFRs promote axonal branch growth and proper branch targeting. Disrupting FGFRs results in miswiring and impaired neural circuit function.
\end{abstract}

\section{Introduction}

Molecular cues allow for axon guidance, target recognition, and synaptogenesis, and along with activity-dependent plasticity, lead to the formation of functioning neural circuits [1]. One molecular strategy used for neuronal wiring is to promote proliferation of neurons that reach their appropriate target using neurotrophic factors [2]. These factors can also induce axonal growth and regulate neuronal survival [3]. Previous studies have demonstrated the strong axonal growth induction potential of neurotrophic factors such as Fibroblast Growth Factor (FGF) $[4,5]$ and Nerve Growth Factor (NGF) in cultured cells $[6,7]$ and animal models [8-10]. However, it is not clear how they might mediate specific axonal targeting decisions that determine neuronal connectivity.

Fibroblast Growth Factors (FGFs) are polypeptides that can be secreted to promote paracrine signaling [11] or act intracellularly as modulators of voltage-gated channels [12]. FGFs function in a wide variety of developmental processes ranging from axis patterning in embryos [13] to induction and maintenance of cell lineages [14]. In humans, the functional diversity of FGFs arises from the combinations of 22 FGFs and four FGF receptors (FGFRs) [15]. In Drosophila

\footnotetext{
* Correspondence: brian.chen@mcgill.ca

${ }^{1}$ Centre for Research in Neuroscience, Research Institute of the McGill University Health Centre, Montréal, Québec, Canada

Departments of Medicine, Neurology and Neurosurgery, McGill University, Montréal, Québec, Canada
}

melanogaster, there are three FGF ligands, Branchless (Bnl), Pyramus (Pyr) and Thisbe (Ths) [16, 17], and two FGFRs, Heartless (Htl) and Breathless (Btl) [18]. Binding of FGF to its receptor promotes dimerization of FGFRs, initiating a molecular cascade that activates phosphatidylinositol 3kinase-Protein Kinase B (PIP3-Akt) and Ras-mitogenactivated protein kinase (MAPK) signaling pathways $[19,20]$. The FGFR signaling pathways are highly conserved in metazoan organisms [21], indicating that FGF and FGFR signaling might function similarly in the neural development of different species.

Vertebrate FGF family members serve a variety of functions throughout neural and synapse development [22]. Vertebrate FGF1 and FGF2 promote neurite outgrowth, whereas FGF2 also contributes to survival of cultured chick neurons [23]. Other FGFs such as FGF7, FGF10, and FGF22, play a role not only in neurite outgrowth but also in presynaptic differentiation [24]. However, it is not known whether FGFs contribute to the specificity of neural wiring. Here we examine FGFR contributions to hard-wired neural circuit formation using the mechanosensory system of Drosophila melanogaster [25, 26]. We find that loss of function of FGFRs in single mechanosensory neurons results in disruptions of synaptic connectivity, including loss of axonal branches. Overexpression of the two Drosophila FGFRs results in targeting errors of ectopic branching. These impairments in mechanosensory 
wiring reduced the animal's ability to respond to mechanical stimuli and elicit a hard-wired cleaning response.

\section{Material and methods Fly stocks}

The following fly stocks were obtained from the Bloomington Drosophila Stock Center: $P\left\{w\left[+m^{*}\right]=n S y b-G A L 4 . S\right\} 3$, $P\{w[+m C]=U A S-b t l . l a m b d a\} 2, \quad P\{w[+m C]=U A S-$ btl.lambda\}1, $P\{w[+m C]=U A S-b t l:: G F P-S 65 T\} 3, P\{y[+t 7.7]$ $v[+t 1.8]=$ TRiP.HMSO2038\}attP2, $\quad P\{y[+t 7.7] \quad v[+t 1.8]=$ TRiP.HMS02656 $\}$ attP40, $\quad P\{y[+t 7.7] \quad v[+t 1.8]=$ TRiP.HMC04140\}attP2, $P\{y[+t 7.7] v[+t 1.8]=$ TRiP.HMS05005 $\}$ attP40, $P\{w[+m C]=$ GAL4-btl.S-hsFLP $\} 2, \quad P\{w[+m C]=U A S p-$ Act5C.T:GFP $\} 2 ; \quad b t l[724] \quad P\{w[+m W . h s]=F R T(w[h s])\} 2 A$ $P\{r y[+t 7.2]=n e o F R T\} 82 B \quad / \quad T M 3, \quad P\{w[+m C]=t u b P-$ GAL80\}3, $\quad P\{w[+m C]=U A S-h t l . M\} Y Y D F R-F 16, \quad P\{y[+t 7.7]$ $v[+t 1.8]=$ TRiP.HMS01437\}attP2, $\quad P\{y[+t 7.7] \quad v[+t 1.8]=$ TRiP.HMJ22375\}attP40, $\quad P\{y[+t 7.7] \quad v[+t 1.8]=$ TRiP.HMS04514\}attP40, P\{y[+t7.7] v[+t1.8] = TRiP.HMS01046\}attP2, $P\{y[+t 7.7] \quad v[+t 1.8]=$ TRiP. HMJ30113 $\}$ attP40, $P\{w[+m C]=$ UAS-htl.DN.M\}33-B40;P\{w[+mC] = UAS-htl.DN.M\}33-B61. The 455-Gal4 line was crossed with UAS-dsRNA Btl, UASdsRNA Bnl, UAS-dsRNA Htl or UAS-dsRNA Pyr to induce RNA interference (RNAi) solely within the scutellum. To overexpress $\mathrm{Btl}$ and $\mathrm{Htl}$, we used 455-Gal4 to selectively drive UAS expression. The $B t l$ rescue line was obtained by crossing UAS-Btl to the respective MARCM $B t l^{\text {null }}$ line while $\mathrm{Htl}$ rescue was obtained by crossing 455-Gal4 / CyO; UAS-Htl / TM6b to Htl DN. DC1.4-Gal4 (a kind gift from Dr. Pat Simpson) was used to selectively express $U A S-$ dsRNA Btl or UAS-Btl in the DorsoCentral neurons [27]. Repo-Gal4 was used to drive UAS expression selectively in glial cells. MARCM $B t l^{\text {null }}$ and $B t l$ rescue lines were heatshocked at $37^{\circ} \mathrm{C}$ for 1 hour at the pupal stage.

\section{Fluorescence in situ hybridization (FISH)}

Fluorescence in situ hybridization for Btl mRNA or $\mathrm{Htl}$ mRNA was performed as described previously [28]. Female adult $\mathrm{nSyb}$-Gal4; UAS-mCD8-GFP fly thoraxes were collected and fixed in $4 \%$ formaldehyde in PBS for $30 \mathrm{~min}$ at room temperature on a shaker, then transferred to cold Dent's fixative (80\% Methanol, 20\% DMSO) and stored at $-20^{\circ} \mathrm{C}$ overnight. The samples were then washed in 100 $\mathrm{mM}$ Tris/ $\mathrm{HCl}$ (pH 7.4), $100 \mathrm{mM} \mathrm{NaCl}$ for $1 \mathrm{~h}$ at room temperature on a shaker, then transferred to $15 \%$ fish gelatin/15\% sucrose for incubation at room temperature overnight on a shaker. Samples were then transferred to $25 \%$ fish gelatin $/ 15 \%$ sucrose and incubated at room temperature overnight on a shaker. Finally, the samples were positioned on their sides in plastic molds containing $20 \%$ fish gelatin $/ 15 \%$ sucrose, flash-frozen with dry ice and stored at $-80^{\circ} \mathrm{C}$. The samples were cut at $10 \mu \mathrm{m}$ thick along the rostral-caudal, dorsal-ventral axis, mounted on slides and dried. Sample outlines were traced with a water repellent pen on the slide. The sections were first repermeabilized with $70 \%$ ethanol for $10 \mathrm{~min}$. Forty-eight custom Stellaris $^{\bullet}$ FISH Probes conjugated to Quasar 570 were designed against $\mathrm{Btl}$ or $\mathrm{Htl}$ mRNA sequence by utilizing the Stellaris ${ }^{\circ}$ RNA FISH Probe Designer version 4.2 (Biosearch Technologies, Inc., Petaluma, CA), available online at www.biosearchtech.com/stellarisdesigner. Probe mix was made by adding the probes to hybridization buffer at a concentration of $125 \mathrm{nM}$, and the probe mix was dispensed onto the slide. The slide was placed in a humidified chamber and incubated for $12 \mathrm{~h}$ overnight in the dark at $37^{\circ} \mathrm{C}$. The probe mix was removed, wash buffer was dispensed on the slide, and incubated in the dark for $30 \mathrm{~min}$ at $37^{\circ} \mathrm{C}$. Two more washes with wash buffer were performed. Finally, the slide was stained and mounted with antifade mountant with DAPI. Fluorescence microscopy was performed using an Olympus laser-scanning confocal microscope FV1000. Images were acquired using a $40 \times$ (N.A. 1.3) or $60 \times$ oil objective (N.A. 1.4).

\section{Carbocyanine dye labeling}

As described previously [26], the morphology of Drosophila pSc mechanosensory axons was visualized via lipophilic carbocyanine dye injection into the open socket of the pSc bristle. We plucked the bristles of two-day old female flies and left them overnight in fixative solution (Paraformaldehyde, $3.7 \%$, in $0.2 \mathrm{M}$ carbonate-bicarbonate buffer at $\mathrm{pH} 9.5$ ). The left and right pSc neurons were labelled using fluorescent carbocyanine dyes, DiI and DiD (Invitrogen ${ }^{\mathrm{Tw}}$, ThermoFisher, Inc.) dissolved in ethanol (final concentration: $20 \mathrm{mg} / \mathrm{mL}$ and $40 \mathrm{mg} / \mathrm{mL}$, respectively). The dye-filled flies were left undisturbed and protected from light in a Petri dish, partially immersed in $0.2 \mathrm{M}$ carbonate-bicarbonate buffer at $\mathrm{pH} 9.5$. After forty-eight hours, the thoracic ganglion was dissected and placed on a slide and imaged under a coverslip.

\section{Image acquisition and analysis}

Fluorescent images were acquired using a Zeiss AxioScope A1 epifluorescence microscope or an Olympus laserscanning confocal microscope FV1000, with a 40x objective (N.A. 1.3). Transmitted light images were acquired and used to evaluate dissection quality and to measure thoracic ganglia width. Only images without CNS damage or surface occlusions were included for data analysis. Maximal intensity projections were stacked from optical sections and adjusted for contrast and brightness if required. Measurements of axonal branch length and branch number were performed blind to genotype. Custom-written software in MATLAB was used to perform the quantitative image analysis.

\section{Behavioral assay and analysis}

The behavioral assay and analysis was performed as previously described [26]. The 455-Gal4 driver was used to selectively express $U A S-d s R N A$ in the scutellum to ensure 
that RNA interference did not perturb the postsynaptic neural circuitry. Two-day old female flies were decapitated and placed in a humidified chamber to recover over a period of 1 hour. After recovery, flies standing on their six legs were tested for responsiveness to stimulation of the anterior notopleural bristle $(\mathrm{aNp})$ with forceps to elicit a cleaning reflex from the anterior legs. Only responsive flies were used for further evaluation by pressure injection of fluorescent dye onto the posterior scutellar bristles. The presence or absence of response was scored visually, with a positive response scored as the successful movement of the rear pair of legs towards the labelled bristles and spread of the dye to the rear legs.

\section{Experimental design and statistical analysis}

In order to reduce the variability due to environmental conditions, age, and sex, flies were reared at $25^{\circ} \mathrm{C}$ on standard cornmeal under $12 \mathrm{~h}$ light/dark cycles and experiments were performed on 2-day old adult female animals. Preliminary unblind experiments were conducted on 10 animals for dye labeling experiments and 30 animals for behavioral assays per genotype to determine adequate sample size through power analysis.

All statistical analysis was performed using SPSS (version 25) and GraphPad Prism (version 7.04). A total of 19 wild-

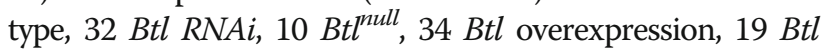
rescue, $20 \mathrm{Bnl}$ RNAi, 14 Pyr RNAi, 38 Htl RNAi, $11 \mathrm{Htl} D N$, $21 \mathrm{Htl}$ overexpression and $10 \mathrm{Htl}$ rescue mutant neurons were analyzed. Shapiro-Wilks test was performed to assess normality on the dataset, and all groups except Btl rescue and $H t l$ overexpression had a $p$ value higher than 0.05 , confirming that the datasets came from Gaussian distributions. One-way ANOVA followed by Dunnett's post hoc pairwise comparison was performed to assess statistical significance in axonal branch length between wildtype and mutant phenotypes. For $B t l$ rescue and $H t l$ overexpression, Mann Whitney $U$ test was performed to assess statistical significance in axonal branch length between wildtype and mutant phenotypes. Kruskal-Wallis non-parametric $H$ test followed by Dunnet's post hoc comparison was performed to assess statistical significance in number of branches between wildtype and mutant phenotypes for the above mentioned groups. Qualitative analysis of axonal variability was performed blind to genotype by randomly distributing the entire axonal arbor dataset. A total of 35 phenotypes were identified among wildtype and mutant axons. These phenotypes were further subdivided into 10 non-mutually exclusive base phenotypes, used for calculation of frequency of targeting errors. Twenty axonal branching patterns were only present in mutant phenotypes (7 Btl RNAi, $9 \mathrm{Btl}$ overexpression, $9 \mathrm{Htl} R \mathrm{RNAi}$ and 5 $\mathrm{Htl}$ overexpression). Quantification of axonal branches and lengths for all genotypes are shown in Additional file 1: Table S1. For the behavioral assay, 116 wildtypes, $113 \mathrm{Btl}$ RNAi, $125 \mathrm{Btl}$ overexpression, $107 \mathrm{Htl}$ RNAi and $76 \mathrm{Htl}$ overexpression flies were tested. To assess association between the wildtype and mutants in the behavioral assay, a $x 2$ test for independence was performed, and all groups had a $p$ value higher than 0.05 , indicating no significant association between the wildtype and mutants. A two-tailed $t$ test for proportions was performed to assess statistical significance in the frequency of response between control and each genotype.

\section{Results}

FGF receptors are expressed in Drosophila mechanosensory neurons

We use the Drosophila mechanosensory system to examine hard-wired synaptic targeting. In this system, each large bristle on the fly notum is innervated by a single mechanosensory neuron whose axon projects within the thoracic ganglion to form specific synaptic connections (Fig. 1). Each mechanosensory axonal arbor is identifiable by its unique and invariant branching pattern. Here we focused our experiments on the left and right posterior scutellar (pSc) neurons due to its accessible anatomical location on the posterior scutellum (Fig. 1a) and its genetic accessibility using the 455-Gal4 driver. First, we sought to determine whether the Btl or Htl FGFRs were transcribed in mechanosensory neurons. We used fluorescent in situ hybridization for detection of $\mathrm{Btl}$ or $\mathrm{Htl}$ mRNA and identified their expression within $\mathrm{pSc}$ neurons (Fig. 1b, c). Interestingly, Btl mRNA was consistently identified in pSc neurons $(n=7)$ and absent in other cell types such as the neighboring glial cell, whereas $H t l$ mRNA $(n=4)$ exhibited a more widespread expression pattern, including within the supporting cells (Fig. 1b, c).

\section{FGFRs are necessary for proper synaptic targeting}

To examine the role of FGFRs in synaptic targeting, we used the invariant axonal branching pattern of $\mathrm{pSc}$ neurons as a readout for axonal targeting errors (Fig. 1d) [28-31]. First, to quantify the baseline variability in wildtype synaptic targeting, we characterized the pSc axonal arbor by measuring the total number of branches and branch lengths in 19 control 455-Gal4/+ animals. A schematic of the control axonal arbor was generated based on the frequency of branch occurrence (Additional file 1: Figure S1), and is consistent with our previous measurements of $\mathrm{pSc}$ neurons in wildtype animals [28, 29] and 455-Gal4 animals [30, 31].

To examine how $B t l$ is used for pSc axonal targeting, we first expressed $d s R N A$ for $B t l$ solely within the pSc neuron using 455-Gal4 driving UAS-dsRNA Btl to induce RNA interference. RNAi knockdown of $B t l$ resulted in disruptions of the pSc axonal branching pattern with noticeable reductions in the number of secondary and tertiary axonal branches (Fig. 1e). To confirm these observations using a genetic knockout of $B t l^{\text {null }}$ within pSc axons, we used the Mosaic Analysis with a Repressible Cell Marker (MARCM) 


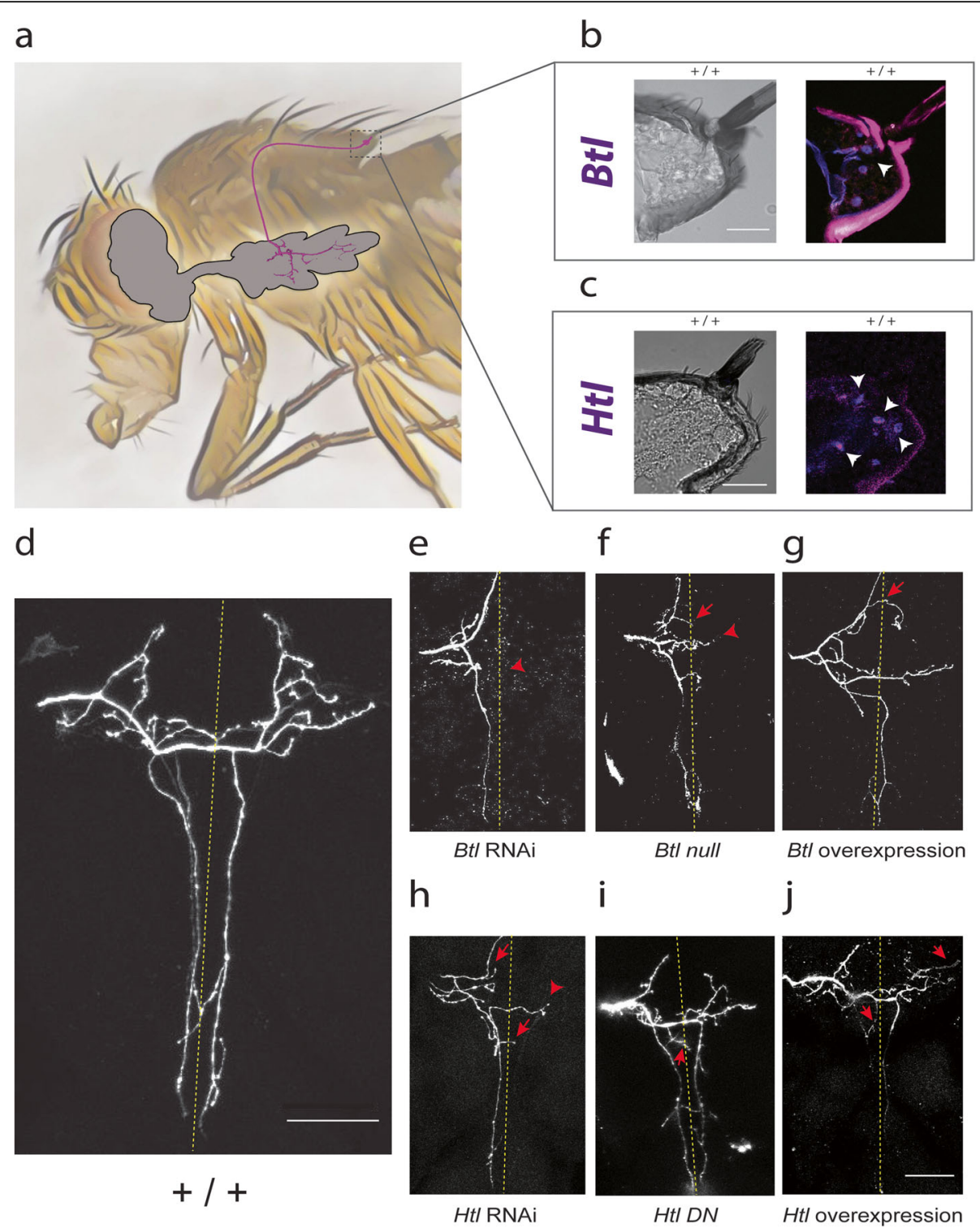

Fig. 1 - The FGFRs Btl and Htl are required for proper synaptic connectivity. a The Drosophila mechanosensory neurons innervate the bristles of the notum. Each bristle is innervated by a single mechanosensory neuron which is uniquely identifiable based on its location. The right pSc neuron and its axonal arbor within the thoracic ganglion are illustrated in magenta. $\mathbf{b}$ A cross section of a pSc bristle is shown in brightfield and the corresponding Btl fluorescence in situ hybridization signal in magenta. Bt/ mRNA (arrowhead) was detected in the neuron underlying the pSc bristle. c A cross section of a pSc bristle is shown with $\mathrm{Ht}$ l fluorescence in situ hybridization signal in magenta (arrowheads). $\mathrm{Htl}$ mRNA was detected in the pSc neuron and its surrounding cells. $\mathbf{d}$ The pSc mechanosensory neuron has a unique and stereotyped axonal branching pattern within the central nervous system. The stereotyped connectivity of the pSc neuron can be used to identify axonal targeting errors in mutants. e-g Mis-regulation of Btl expression results in synaptic targeting errors. e Knockdown of Btl using RNAi resulted in a loss of core branches. $\mathbf{f} B t{ }^{\text {pull }}$ MARCM mutants also had missing core branches (arrowhead), mostly contralateral to the axonal entry site into the thoracic ganglion. $\mathbf{g}$ Increasing Btl expression resulted in misrouting errors as well as ectopic branch formations. $\mathbf{h}$-j Mis-regulation of $\mathrm{Htl}$ expression also results in targeting errors. $\mathbf{h}$ Knockdown of $\mathrm{Htl}$ using RNAi resulted in ectopic branches (arrows) and loss of secondary branches (arrowhead). $\mathbf{i} \mathrm{Htl}$ Dominant Negative ( $\mathrm{Htl}$ DN) mutants showed similar axonal targeting errors as $\mathrm{Htl}$ RNAi mutants such as ectopic branches towards the midline (arrow). j Overexpression of $\mathrm{Htl}$ also resulted in ectopic branches (arrows). Scale bars, $20 \mu \mathrm{m}$ in (b, $\mathbf{c}), 50 \mu \mathrm{m}$ in $(\mathbf{d}, \mathbf{j})$. Arrowheads point to absent branches, arrows point to ectopic branches

technique to produce homozygous Btl loss of function within only a few cells in the mosaic animal (Fig. 1f). We found that the homozygous $B t l^{\text {null }} \mathrm{pSc}$ axons in these MARCM flies phenocopied the RNAi knockdown of $B t l$ $(n=10$ and 32 , respectively), displaying a noticeable decrease in branching complexity and loss of branches. Whereas Btl knockdown and loss of function axonal arbors displayed a reduction of secondary and tertiary branches, overexpression of $B t l$ within pSc neurons (455-Gal4 / $U A S-B t l, n=34$ ) resulted in a pronounced increase in the 
number of ectopic branches (Fig. 1g). Importantly, none of these experimental manipulations altered the axon guidance of the $\mathrm{pSc}$ axon from the periphery into the central nervous system via the mesothoracic root.

Is the axonal branch growth-promoting function of Btl shared with the other FGFR Htl? We evaluated the axonal arbors of pSc neurons in different $\mathrm{Htl}$ mutant animals (Fig. 1h-j). Htl RNAi mutants (455-Gal4 / UASdsRNA $\mathrm{Htl}$ ) showed an increased frequency of ectopic branches anteriorly while they also had a significant reduction of secondary and tertiary branches (Fig. 1h). To verify these RNAi results, we analyzed the axonal arbors of $\mathrm{pSc}$ neurons overexpressing Htl Dominant Negative, which disrupts Htl protein function (455-Gal4; UAS-Htl DN) (Fig. 1i). We observed that Htl DN mutants displayed a partial phenocopy of $H t l$ RNAi targeting errors $(n=11$ and 38, respectively), including the presence of ectopic branches in anterior region of the thoracic ganglion. Similar to Btl overexpression mutants, $\mathrm{Htl}$ overexpression mutants showed an increase in the number of ectopic branches (Fig. 1j).

Quantitative analysis showed a significant difference $(F$ $(3.92)=4.732, p=0.0041)$ in total axonal branch lengths between control, $B t l$ RNAi, $B t l^{\text {null }}$ MARCM and $B t l$ overexpression (Fig. 2). Compared to control animals (Fig. 2a), Btl RNAi and $B t l^{\text {null }}$ (Fig. 2b, c, respectively) neurons showed a significant decrease $(n=32, p=0.0067$ and $n=10, p<$ 0.0001 , respectively) in total axonal branch lengths. Conversely, $B t l$ overexpression (Fig. 2d) resulted in a significant increase $(n=34, p=0.0031)$ in total branch length compared to wildtype. In Btl RNAi and Btt ${ }^{\text {null }}$, the decrease in arbor complexity was mostly due to decreased lengths of secondary and tertiary branches rather than arbors with less branches (Fig. 2f-h). Axonal arbors with ectopic branches were more frequently observed in Btl overexpression, resulting in a significant increase in the total number of branches $(p=0.0057)$ compared to controls (Fig. 2g). For $B t l$ rescue experiments $(n=19)$ (Fig. 2e), we observed no significant differences in total branch length or branch number compared to controls.

Statistical analysis of $\mathrm{Htl}$ mutants showed a significant difference $(F(3.73)=3.154, p=0.0299)$ in total axonal branch lengths between controls, $H t l$ RNAi, $H t l D N$ and $H t l$ rescue (Fig. 3). Neurons expressing $H t l$ RNAi (Fig. 3b) showed a significant decrease $(n=38, p=0.0161)$ in total axonal branch lengths, whereas overexpression of $\mathrm{Htl}$ (Fig. 3d) resulted in a significant increase in total branch length ( $n=21, p<0.0001)$ (Fig. 3f-h). Htl RNAi mutants also had significantly fewer secondary and tertiary core branches $(p=0.0046)$ in addition to reduced branch lengths, resulting in an overall reduction in axonal arbor complexity compared to control neurons (Fig. 3f-h). Overexpression of $\mathrm{Htl}$ in pSc axons resulted in a significant increase in total number of branches $(n=21, p=0.0185$, Fig. 3d) due to a large number of ectopic branch formations. For $\mathrm{Htl} \mathrm{DN}(n=11)$ and $\mathrm{Htl}$ rescue $(n=10)$ experiments (Fig. $3 \mathrm{c}$ and e, respectively), there were no significant differences in total branch length or branch number.

Both FGFRs $B t l$ and $H t l$ were required for proper branch routing as well. Loss of function and overexpression mutants for both genes displayed stereotyped errors related to inappropriate axonal branch routing. Midline crossing errors were more frequently observed in overexpression mutants (representative examples in Figs. 1g, 2d, 3d), while branch misrouting was equally observed among knockdown and overexpression mutants (Fig. 2b). Frequency of midline crossing errors and ectopic branches for all genotypes are shown in Additional file 1: Table S1.

\section{Disruption of $B t /$ and $H t l$ expression in the $\mathrm{pSc}$ axon impairs the cleaning reflex}

We next investigated whether the observed disruptions in pSc synaptic connectivity affected neural circuit function by testing the behavioral response of FGFR mutant mosaic animals. In wildtype animals, mechanical stimulation of the pSc bristle evokes a cleaning reflex, where the third pair of legs grooms the bristle in an attempt to end the stimulus (Fig. 4a). We used the 455-Gal4 driver to knock down or overexpress $\mathrm{Btl}$ and $\mathrm{Htl}$ within the $\mathrm{pSc}$ neuron without affecting the downstream mechanosensory circuitry. We found that all mutant groups displayed a significantly reduced response rate $(p<0.01)$ in the cleaning reflex when compared to controls (Fig. 4b).

To determine if the presence of targeting errors would lead to a lower response rate, we analyzed the axonal arbors of animals with a positive grooming reflex (responders) and animals without a grooming reflex response (non-responders) in Btl RNAi mutants (Fig. 4c). Quantitative analysis revealed a significant difference $(F(2,42)=4.158$, $p=0.0052)$ between the control animals, Btl RNAi responders and $B t l$ RNAi non-responders. Btl RNAi responders and non-responders showed a significant decrease ( $n=13, p=0.0062$ and $n=10, p=0.0244$, respectively) in total branch lengths (Fig. 4d) and branch numbers $(p<$ 0.0001 and $p=0.0036$, respectively) (Fig. 4e) compared to controls.

\section{Knockdown of FGFs, Bnl and Pyr, partially phenocopies FGFR mutants}

To verify the signaling mechanism by which the FGFRs control axonal branch growth, we performed RNAi knockdown experiments on the FGFs $B n l$ and $P y r$, the ligands for $B t l$ and $H t l$, respectively. Because FGF/FGFR signaling can act in an autocrine and paracrine manner and it is not known which cells might express the FGF ligands, we used the 455Gal4 driver to express the dsRNA for Bnl and Pyr in the primordial scutellum (Fig. 5). 455-Gal4 is active in the larval imaginal discs and persists throughout pupal development, being expressed in all cells that give rise to the scutellum, 


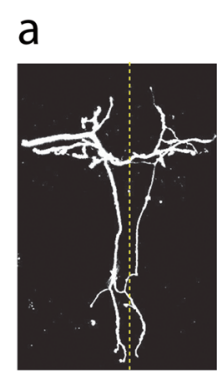

Control

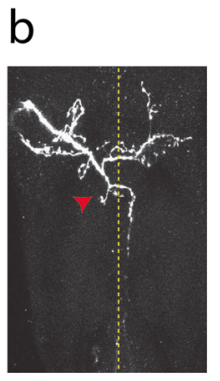

Btl RNAi

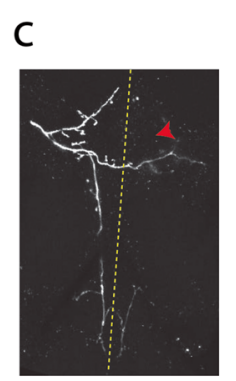

BtI null d

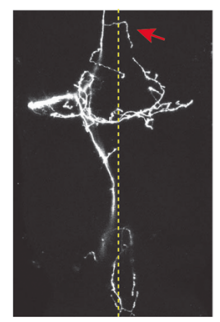

$B t /$ overexpression

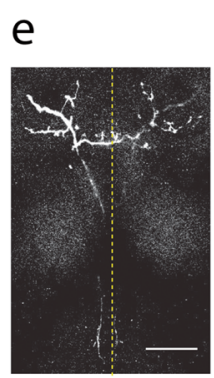

$B t /$ rescue f

Less Branches $\quad+1+\quad$ More Branches

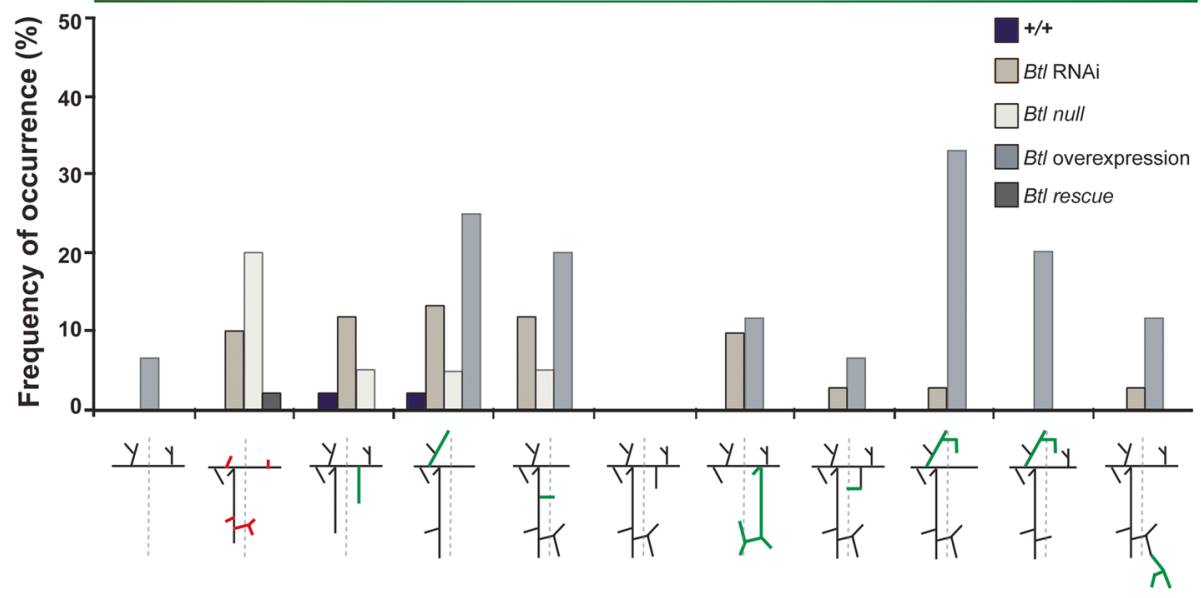

g

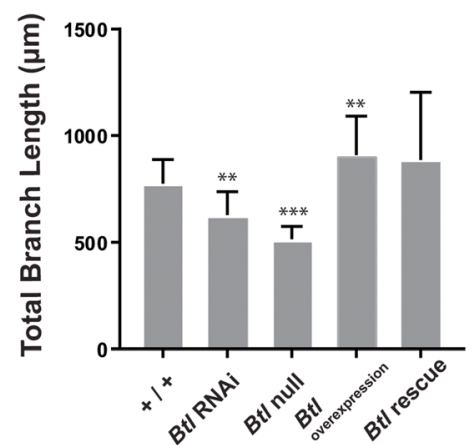

h

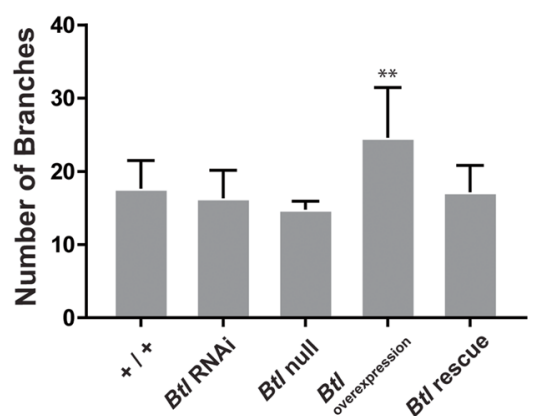

Fig. 2 - Altered Bt/ expression results in axonal branch growth errors and misrouting. a-d Disrupted Bt/ expression produced a higher frequency of axonal branching errors. Expression of Bt/ dsRNA solely within the pSc neuron had a higher frequency of routing errors and absence of core

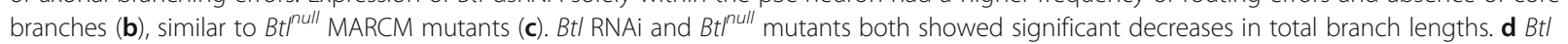
overexpression resulted in an increase in total branch length and significantly more ectopic axonal branches and routing errors. e Rescue expression of Btl in MARCM mutants reverted the axonal branching patterns to wildtype. Red indicates reduction of branches, green indicates ectopic branches. $\mathbf{f} B t$ l loss of function versus overexpression had opposing effects on axonal branch length $(\mathbf{g})$ and number of branches (h). Scale bar, 50 mm. Arrowheads point to absent branches, arrows point to ectopic branches. Error bars are S.D.

including the epithelial cells and sensory organ precursors that will become the pSc neuron, pSc glial, pSc socket cell, and other surrounding cells that create the pSc bristle structure. We found that the axonal arbors of 455-Gal4 / UASdsRNA Bnl animals had five axonal targeting error patterns that were also present in Btl RNAi mutants (Fig. 5c, d), of which the frequency of occurrence for two targeting error types were significantly higher $(p=0.0466)$ in $B n l$ RNAi mutants $(n=20)$ than Btl RNAi mutants $(n=32)$ (Fig. 5g). In Pyr RNAi mutants, we also observed five targeting error types that were also found in $H t l$ RNAi mutants (Fig. 5e, f). However, the frequency of occurrence of these phenocopies did not differ significantly between Pyr RNAi and $H t l$ RNAi mutants (Fig. 5h). 
a

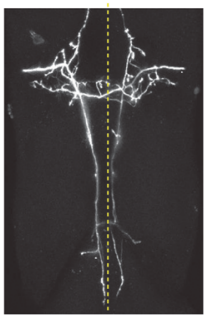

f b

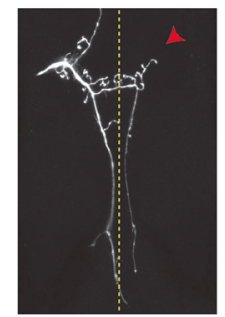

HtI RNAi
C

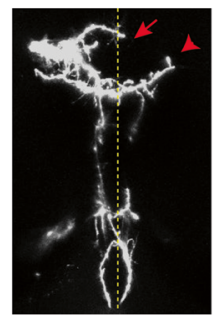

$H t I D N$

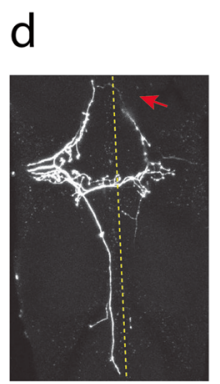

$H t$ l overexpression e

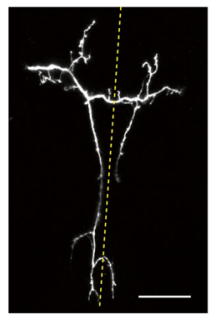

$H t /$ rescue

$\begin{array}{lll}\text { Less Branches } & +l+ & \text { More Branches }\end{array}$

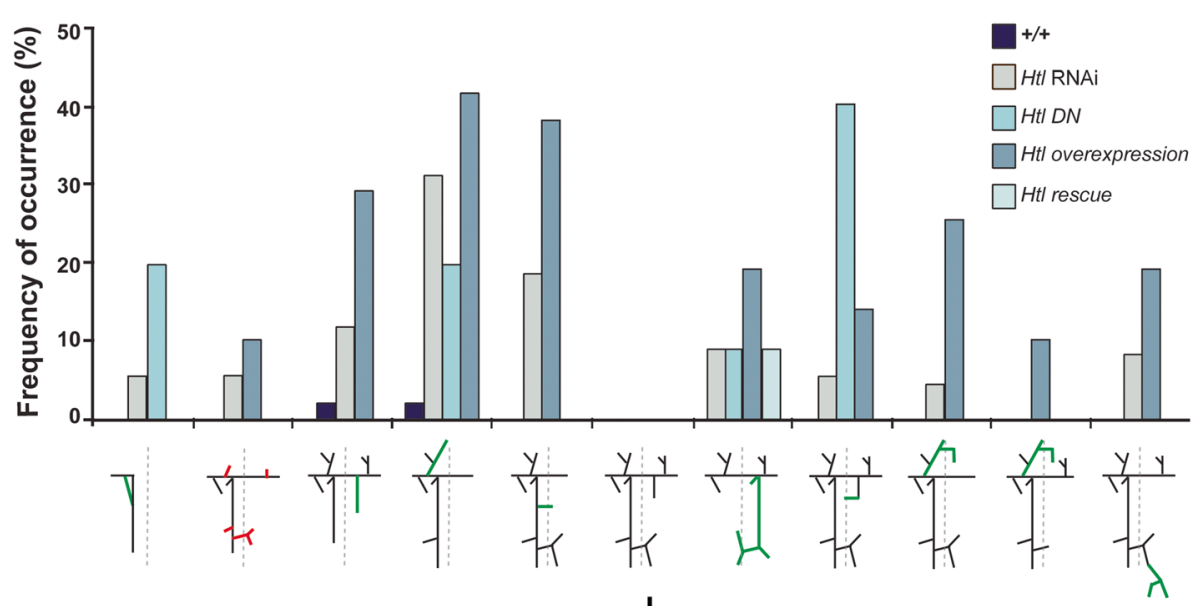

g

h
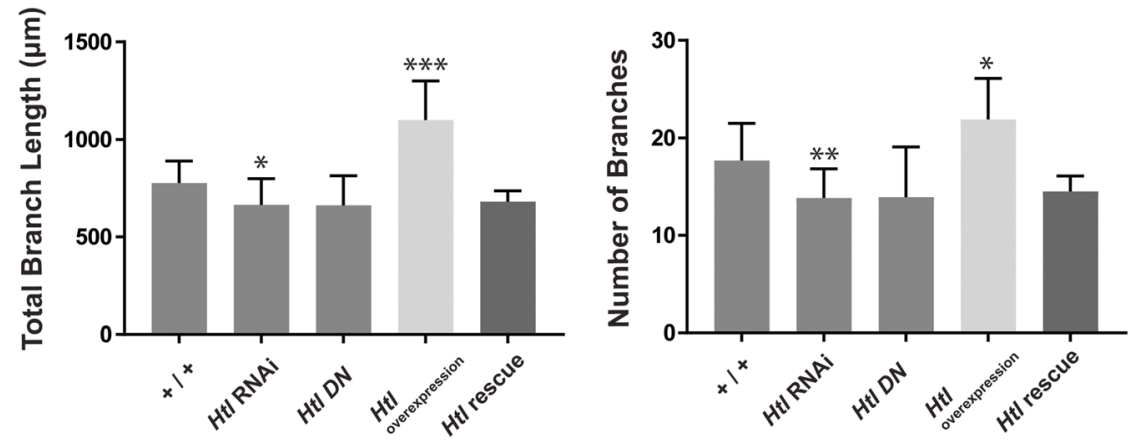

Fig. 3 - Altered expression of $\mathrm{Ht}$ l disrupts pSc neuron synaptic connectivity. a-d $\mathrm{Ht} /$ mutants have a higher frequency of synaptic targeting errors. Representative examples of wildtype pSc axonal arbors (a), Htl RNAi (b), Htl Dominant Negative (c) and Htl overexpression (d). e Overexpression of $\mathrm{Ht}$ l with the $\mathrm{Htl} \mathrm{DN}$ in the $\mathrm{pSc}$ neuron reverted the axonal branching pattern to wildtype. $\mathbf{f} \mathrm{Htl}$ mutants had stereotyped pSc axonal targeting errors, similar to Bt/ mutants. Red indicates reduction of branches, green indicates ectopic branches. Quantitative analysis showed a significant decrease in the total branch length $(\mathbf{g})$ and total number of branches (h) in Htl RNAi compared to control arbors. In contrast, Htl overexpression within the pSc neuron resulted in a higher occurrence of axonal branch misrouting and ectopic branches, but also missing core branches (f). Overall, $\mathrm{Htl}$ overexpression resulted in a significant increase in total branch length $(\mathbf{g})$ and total number of branches $(\mathbf{h})$ compared to control. Scale bar is $50 \mu \mathrm{m}$. Arrowheads point to absent branches, arrows point to ectopic branches. Error bars are S.D.

\section{Discussion}

In insects, FGFRs modulate gene expression, cellular interactions, and cellular morphology [13, 20, 32]. During Drosophila organogenesis, FGF ligands promote dorsal migration of ectoderm and radial movement of neuroectoderm [16, 33, 34]. The $H t l$ FGFR regulates cellular migration within the mesoderm during embryonic development [35]. The Btl FGFR plays a central role in the development of the Drosophila respiratory system by inducing tracheal tube branching [36, 37]. Our results add a novel function to Drosophila FGFRs in axonal arbor formation. We found that downregulation of $\mathrm{Btl}$ or $\mathrm{Htl}$ 
a

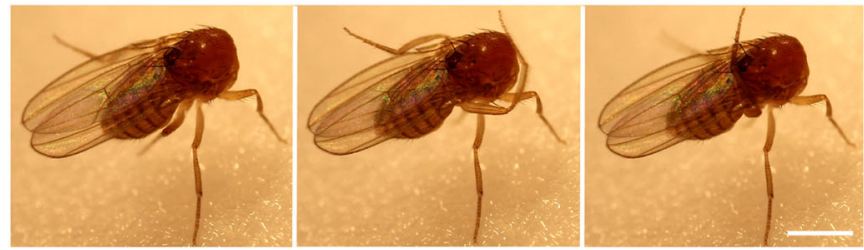

b

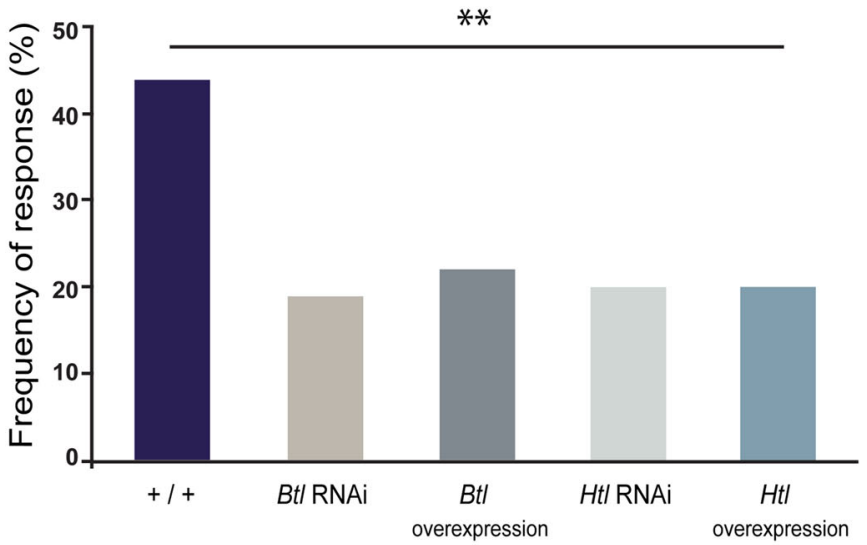

C

BtI RNAi Bt/ RNAi
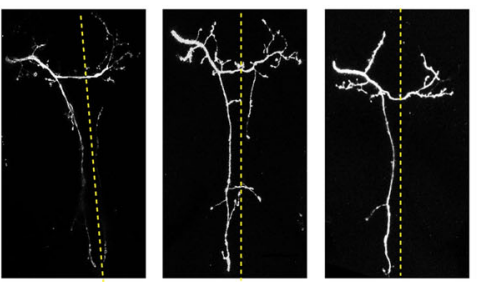

Btl overexpression Btl overexpression

no response

response

d

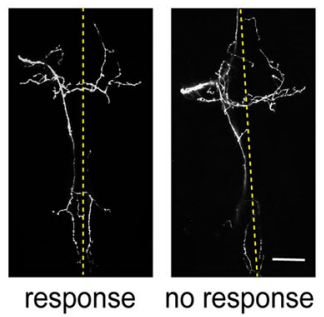

e
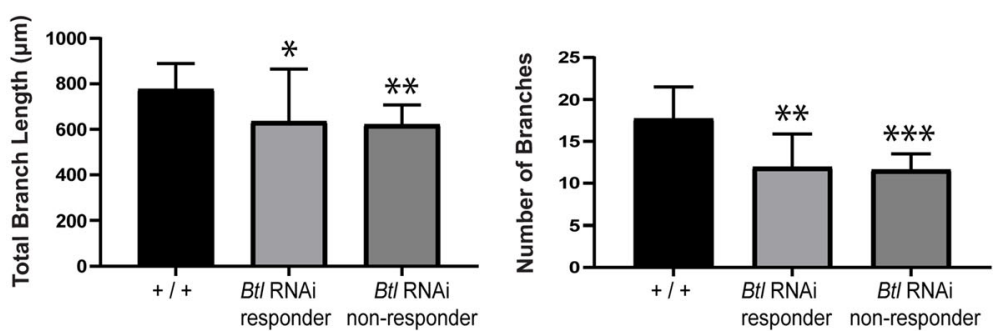

Fig. 4 - Axonal targeting errors impair neuronal connectivity and mechanosensation. a Stimulation of the pSc bristle via application of fluorescent dye triggers a grooming response. A positive response was visually scored as the successful movement of the rear pair of legs onto the labelled bristles with spread of the dye to the rear legs. Scale bar is $1 \mathrm{~mm}$. b, c Disrupting the pSc connectivity led to a significant decrease $(p<0.01)$ in response rate of all Btl and $\mathrm{Htl}$ mutant groups when compared to control. c Direct correlation of the pSc axonal branching pattern with the animal's behavioral response revealed that animals with more severe axonal targeting errors ("no response", far left and far right images) were more likely to be non-responsive to mechanical stimulation. Scale bar is $50 \mu \mathrm{m}$. Total branch lengths (d) and branch numbers (e) were significantly smaller in Bt/ RNAi responders $(p<0.05)$ and Bt/ RNAi non-responders $(p<0.01)$ compared to controls. Error bars are S.D.

expression resulted in loss of axonal arbor size and number of branches, whereas overexpression of FGFRs resulted in more frequent routing errors and ectopic branches. These disruptions of synaptic connectivity from FGFR misexpression reduced the animal's behavioral responses, highlighting the importance and sensitivity of the 


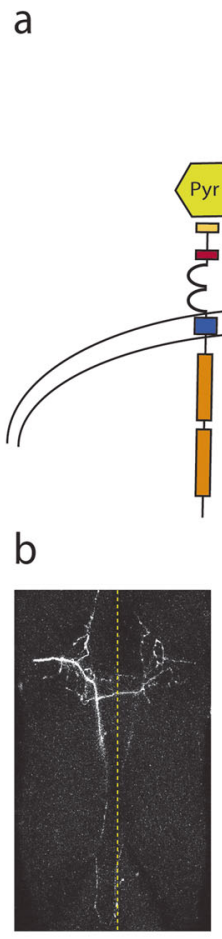

control

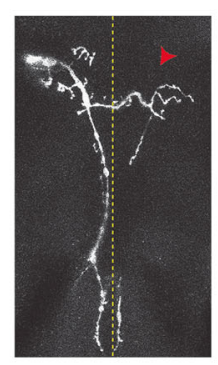

Btl RNAi

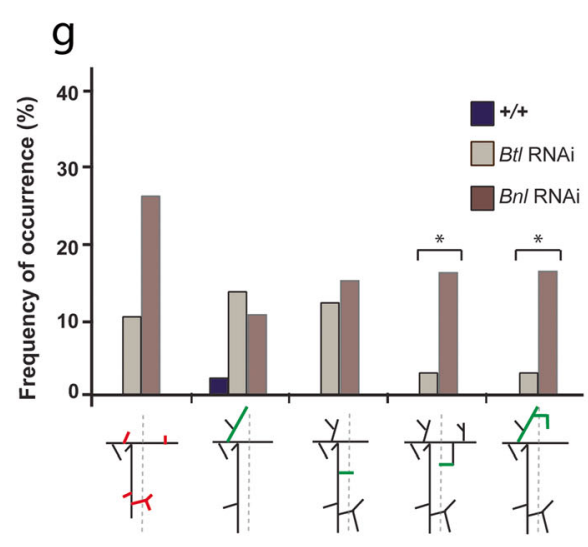

d

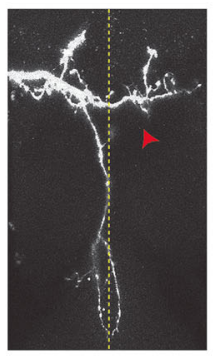

BnI RNAi

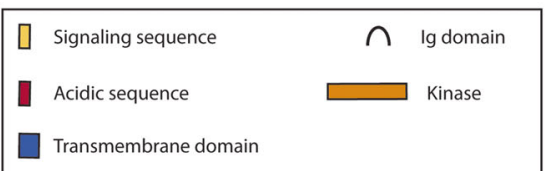

e

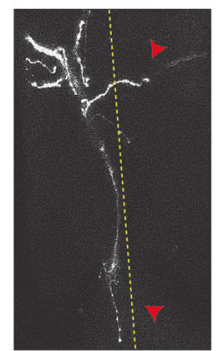

$H t$ RNAi f

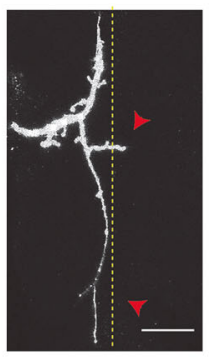

Pyr RNAi

$\mathrm{h}$

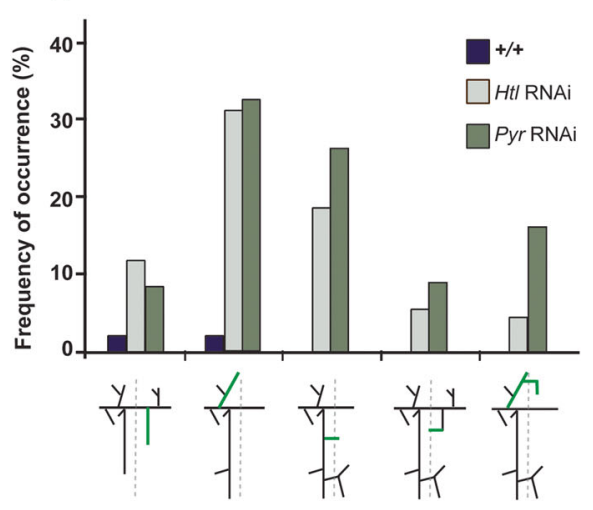

Fig. 5 - Specific axonal targeting errors are phenocopied in FGF ligand and FGFR mutants. a The FGF ligands Bnl and Pyr bind to their respective FGFRs Btl and $\mathrm{Htl}$ at the cell surface. b-f Representative examples of a wildtype axonal arbor (b), Bt/ RNAi (c), Bn/ RNAi (d), Htl RNAi (e) and Pyr RNAi (f) are shown. $\mathbf{g}$ Five error types had significantly greater frequency of occurrence in both Bt/ RNAi and Bn/ RNAi mutants compared to wildtype flies. Two targeting error phenotypes had a significantly higher frequency of occurrence $(p<0.05)$ in the Bn/ RNAi mutants than in Btl RNAi mutants. $\mathbf{h}$ Pyr RNAi mutants also had five error types that occurred at significantly greater frequency than in wildtype flies that were also found in $\mathrm{Htl}$ RNAi mutants. Scale bar is $50 \mu \mathrm{m}$. Arrowheads point to absent branches, arrows point to ectopic branches

pSc neuron connectivity to proper circuit function, and the sensitivity to FGFR expression in synaptic targeting. In Drosophila, both FGFRs $\mathrm{Btl}$ and $\mathrm{Htl}$ activate similar pathways with only a small variation in protein affinity [20, 38]. FGFR functional redundancy could explain the similar effects of overexpression and knockdown of Btl and $\mathrm{Htl}$ in our results.

The axonal outgrowth and branching functions of FGF ligands have also been observed in cell culture in the mammalian nervous system [4, 5, 9]. Previous studies have also demonstrated the contributions of FGFR signaling to other targeting molecules, such as slit1 [39]. In our study, we demonstrate that the axonal branch outgrowth and targeting function of FGFRs is critical for proper synaptic connectivity and circuit function. Furthermore, the stereotyped $\mathrm{pSc}$ axonal targeting errors that occurred in Btl and $\mathrm{Htl}$ mutants (Figs. 2f, 3f) suggest that FGFRs are involved in recognizing specific axonal or synaptic targets at distinct 
branching points or extracellular locations, where loss or gain of FGFR function results in specific misrouting errors.

The axonal outgrowth function of FGFR may be present in all mechanosensory neurons. Using a different Gal4 driver, DC1.4-Gal4, which is specific for DorsoCentral mechanosensory neurons, we expressed dsRNA Btl $(n=6)$ and observed a loss of some axonal branches (Additional file 1: Figure S2). Overexpression of $B t l(n=10)$ resulted in excess branching along the midline. However, the extent to which FGFRs are expressed in other mechanosensory neurons is not known.

FGF signaling has also been implicated in glial migration, differentiation and formation of the neuronal sheath in Drosophila [40, 41]. FGFs can control neural circuit formation through epidermal-glial and neuronal-glial interactions to specify glia, and subsequently, synapse location [42]. However, a glial source of FGFs did not seem to influence pSc axonal targeting when the pan-glial driver repo-Gal4 was used to knock down the FGFs Bnl or Pyr (Additional file 1: Figure S3). These pan-glial RNAi Bnl and RNAi Pyr animals were very weak and developmentally delayed, making the results difficult to interpret. Thus, the cellular source of FGF ligands and the cellular mechanisms of FGFR activation and signaling in mechanosensory neurons remain unknown. Further examinations of the relationships and expression patterns of the FGF ligands $B n l, P y r$, and Ths and their FGFRs $\mathrm{Btl}$ and $\mathrm{Htl}$ within neurons and their target cells will help elucidate the roles of FGF signaling in axonal outgrowth and synaptic targeting.

\section{Supplementary information}

Supplementary information accompanies this paper at https://doi.org/10 1186/s13041-019-0503-y.

Additional file 1: Table S1. Quantitative analysis of axonal arbors for each genotype. Figure S1. A schematic of the wildtype pSc axonal arbor was established based on branch size and location. Figure S2. Bt/ is required for proper axonal targeting in the pDc mechanosensory neuron. Figure S3. FGF ligands and FGFRs genetic analyses in glial cells.

\section{Acknowledgments}

The authors thank Farida Emran and Isabela Fabri Karam for assistance with experiments and analysis.

\section{Authors' contributions}

B.E.C. designed the experiments and supervised the project. J.V.S., R.Y.Y., and A.A.T.O. performed experiments and analyzed the data. J.V.S. and B.E.C. wrote the manuscript. All authors read and approved the final manuscript.

\section{Funding}

This work was supported by grants (to B.E.C.) from the Natural Sciences and Engineering Research Council of Canada, and the Canadian Institutes of Health Research (185452). The funding agencies had no role in the design of the study and collection, analysis, and interpretation of data and in writing the manuscript.

\section{Availability of data and materials}

The datasets, analyses, and materials used in the current study are available from the corresponding author on reasonable request.
Ethics approval and consent to participate

Not Applicable.

\section{Consent for publication}

Not Applicable.

\section{Competing interests}

The authors declare that they have no competing interests.

Received: 30 April 2019 Accepted: 1 October 2019

Published online: 24 October 2019

\section{References}

1. Li M, Liu J, Tsien JZ. Theory of connectivity: nature and nurture of cell assemblies and cognitive computation. Front Neural Circuits. 2016;10:34.

2. Aloe L, Rocco M, Bianchi P, Manni L. Nerve growth factor: from the early discoveries to the potential clinical use. J Transl Med. 2012;10(1):239.

3. Keefe K, Sheikh I, Smith G. Targeting Neurotrophins to specific populations of neurons: NGF, BDNF, and NT-3 and their relevance for treatment of spinal cord injury. Int J Mol Sci. 2017;18(3):548.

4. Szebenyi G, Dent EW, Callaway JL, Seys C, Lueth H, Kalil K. Fibroblast growth factor-2 promotes axon branching of cortical neurons by influencing morphology and behavior of the primary growth cone. J Neurosci. 2001; 21(11):3932-41.

5. Klimaschewski L, Nindl W, Feurle J, Kavakebi P, Kostron H. Basic fibroblast growth factor isoforms promote axonal elongation and branching of adult sensory neurons in vitro. Neuroscience. 2004;126(2):347-53.

6. Colombo F, Racchetti G, Meldolesi J. Neurite outgrowth induced by NGF or L1CAM via activation of the TrkA receptor is sustained also by the exocytosis of enlargeosomes. Proc Natl Acad Sci. 2014;111(47):16943-8.

7. Hausott B, Rietzler A, Vallant N, Auer M, Haller I, Perkhofer S, Klimaschewski L. Inhibition of fibroblast growth factor receptor 1 endocytosis promotes axonal branching of adult sensory neurons. Neuroscience. 2011;188:13-22.

8. Li W, Shi L, You Y, Gong Y, Yin B, Yuan J, Peng X. Downstream of tyrosine kinase/docking protein 6, as a novel substrate of tropomyosin-related kinase $\mathrm{C}$ receptor, is involved in neurotrophin 3-mediated neurite outgrowth in mouse cortex neurons. BMC Biol. 2010;8(1):86.

9. Shimada T, Yoshida T, Yamagata K. Neuritin mediates activity-dependent axonal branch formation in part via FGF signaling. J Neurosci. 2016;36(16):4534-48.

10. McFarlane S, McNeill L, Holt CE. FGF signaling and target recognition in the developing Xenopus visual system. Neuron. 1995;15(5):1017-28.

11. Hannan RL, Kourembanas S, Flanders KC, Rogelj SJ, Roberts AB, Faller DV, Klagsbrun M. Endothelial cells synthesize basic fibroblast growth factor and transforming growth factor Beta. Growth Factors. 1988:1 (1):7-17.

12. Lou J-Y, Laezza F, Gerber BR, Xiao M, Yamada KA, Hartmann H, Craig AM, Nerbonne JM, Ornitz DM. Fibroblast growth factor 14 is an intracellular modulator of voltage-gated sodium channels. J Physiol. 2005;569(1):179-93.

13. Hashiguchi M, Mullins MC. Anteroposterior and dorsoventral patterning are coordinated by an identical patterning clock. Development. 2013;140(9):1970-80.

14. Kang W, Hebert JM. FGF signaling is necessary for neurogenesis in young mice and sufficient to reverse its decline in old mice. J Neurosci. 2015; 35(28):10217-23.

15. Zhang $X$, Ibrahimi OA, Olsen SK, Umemori H, Mohammadi M, Ornitz DM. Receptor specificity of the fibroblast growth factor family. J Biol Chem. 2006; 281(23):15694-700.

16. Stathopoulos A. Pyramus and thisbe: FGF genes that pattern the mesoderm of Drosophila embryos. Genes Dev. 2004:18(6):687-99.

17. Kadam S, McMahon A, Tzou P, Stathopoulos A. FGF ligands in Drosophila have distinct activities required to support cell migration and differentiation. Development. 2009;136(5):739-47.

18. Muha V, Müller H-A. Functions and mechanisms of fibroblast growth factor (FGF) Signalling in Drosophila melanogaster. Int J Mol Sci. 2013;14(3):5920-37.

19. Dailey L, Ambrosetti D, Mansukhani A, Basilico C. Mechanisms underlying differential responses to FGF signaling. Cytokine Growth Factor Rev. 2005; 16(2):233-47.

20. Ornitz DM, Itoh $N$. The fibroblast growth factor signaling pathway. Wiley Interdiscip Rev Dev Biol. 2015;4(3):215-66.

21. Itoh N, Ornitz DM. Evolution of the Fgf and Fgfr gene families. Trends Genet. 2004;20(11):563-9. 
22. Dono R. Fibroblast growth factors as regulators of central nervous system development and function. Am J Phys Regul Integr Comp Phys. 2003;284(4):R867-81.

23. Pataky DM, Borisoff JF, Fernandes KJL, Tetzlaff W, Steeves JD. Fibroblast growth factor treatment produces differential effects on survival and Neurite outgrowth from identified Bulbospinal neurons in vitro. Exp Neurol. 2000;163(2):357-72.

24. Umemori H, Linhoff MW, Ornitz DM, Sanes JR. FGF22 and its close relatives are presynaptic organizing molecules in the mammalian brain. Cell. 2004; 118(2):257-70.

25. Ghysen A. The projection of sensory neurons in the central nervous system of Drosophila: choice of the appropriate pathway. Dev Biol. 1980;78(2):521-41.

26. Kays I, Cvetkovska V, Chen BE. Structural and functional analysis of single neurons to correlate synaptic connectivity with grooming behavior. Nat Protoc. 2013:9(1):1-10.

27. Garcia-Garcia MJ, Ramain P, Simpson P, Modolell J. Different contributions of pannier and wingless to the patterning of the dorsal mesothorax of Drosophila. Development. 1999;126(16):3523-32.

28. Cvetkovska V, Hibbert AD, Emran F, Chen BE. Overexpression of Down syndrome cell adhesion molecule impairs precise synaptic targeting. Nat Neurosci. 2013;16(6):677-82.

29. Chen Brian E, Kondo M, Garnier A, Watson F, Puettmann-Holgado R, Lamar $D$, Schmucker D. The molecular diversity of Dscam is functionally required for neuronal wiring specificity in Drosophila. Cell. 2006;125(3):607-20.

30. Neufeld SQ, Hibbert AD, Chen BE. Opposing roles of PlexinA and PlexinB in axonal branch and varicosity formation. Mol Brain. 2011;4:15.

31. Kays I, Cvetkovska V, Chen Brian E. Structural and functional analysis of single neurons to correlate synaptic connectivity with grooming behavior. Nat Protoc. 2014;9(1):1-10.

32. Ornitz DM, Itoh N. Fibroblast growth factors. Genome Biol. 2001;2(3): REVIEWS3005.

33. Klingseisen A, Clark IBN, Gryzik T, Muller HAJ. Differential and overlapping functions of two closely related Drosophila FGF8-like growth factors in mesoderm development. Development. 2009;136(14):2393-402.

34. Du L, Zhou A, Patel A, Rao M, Anderson K, Roy S. Unique patterns of organization and migration of FGF-expressing cells during Drosophila morphogenesis. Dev Biol. 2017;427(1):35-48.

35. Murray MJ, Saint R. Photoactivatable GFP resolves Drosophila mesoderm migration behaviour. Development. 2007;134(22):3975-83.

36. Metzger RJ. Genetic control of branching morphogenesis. Science. 1999 284(5420):1635-9.

37. Lubarsky B, Krasnow MA. Tube Morphogenesis. Cell. 2003;112(1):19-28

38. Ornitz DM. FGFs, heparan sulfate and FGFRs: complex interactions essential for development. BioEssays. 2000;22(2):108-12

39. Yang JJ, Bertolesi GE, Hehr CL, Johnston J, McFarlane S. Fibroblast growth factor receptor 1 signaling transcriptionally regulates the axon guidance cue slit1. Cell Mol Life Sci. 2018;75(19):3649-61.

40. Guillemot F, Zimmer C. From cradle to grave: the multiple roles of fibroblast growth factors in neural development. Neuron. 2011;71(4):574-88.

41. Franzdóttir SR, Engelen D, Yuva-Aydemir Y, Schmidt I, Aho A, Klämbt C. Switch in FGF signalling initiates glial differentiation in the Drosophila eye. Nature. 2009.

42. Shao Z, Watanabe S, Christensen R, Jorgensen Erik M, Colón-Ramos Daniel A. Synapse location during growth depends on glia location. Cell. 2013; 154(2):337-50.

\section{Publisher's Note}

Springer Nature remains neutral with regard to jurisdictional claims in published maps and institutional affiliations.

Ready to submit your research? Choose BMC and benefit from:

- fast, convenient online submission

- thorough peer review by experienced researchers in your field

- rapid publication on acceptance

- support for research data, including large and complex data types

- gold Open Access which fosters wider collaboration and increased citations

- maximum visibility for your research: over $100 \mathrm{M}$ website views per year

At $\mathrm{BMC}$, research is always in progress.

Learn more biomedcentral.com/submissions 